\title{
Simultaneous destabilization of $\beta$-catenin and Ras via targeting of the axin-RGS domain as a potential therapeutic strategy for colorectal cancer
}

\author{
Pu-Hyeon Cha ${ }^{1,2}$ E Kang-Yell Choi, ${ }^{1,2}$ \\ ${ }^{1}$ Translational Research Center for Protein Function Control, Yonsei University, ${ }^{2}$ Department of Biotechnology, College of Life Science and \\ Biotechnology, Yonsei University, Seoul 03722, Korea
}

\begin{abstract}
Mutations of $A P C$ and $K R A S$ are frequently observed in human colorectal cancers (CRCs) and the Wnt/ $\beta$-catenin and Ras pathways are consequently activated in a significant proportion of CRC patients. Mutations in these two genes are also known to synergistically induce progression of CRCs. Through a series of studies, we have demonstrated that inhibition of the Wnt/ $\beta$-catenin signaling pathway negatively regulates Ras stability, therefore, Ras abundance is increased together with $\beta$-catenin in both mice and human CRCs harboring adenomatous polyposis coli (APC) mutations. In a recent study, we identified KY1220, a small molecule that simultaneously degrades $\beta$-catenin and Ras by inhibition of the $\mathrm{Wnt} / \beta$-catenin pathway, and obtained its derivative KYA1797K, which has improved activity and solubility. We found that KYA1797K binds the RGS domain of axin and enhances the binding affinity of $\beta$-catenin or Ras with the $\beta$-catenin destruction complex components, leading to simultaneous destabilization of $\beta$-catenin and Ras via GSK3 $\beta$ activation. By using both in vitro and in vivo studies, we showed that KYA1797K suppressed the growth of CRCs harboring APC and KRAS mutations through destabilization of $\beta$-catenin and Ras. Therefore, our findings indicate that the simultaneous destabilization of $\beta$-catenin and Ras via targeting axin may serve as an effective strategy for inhibition of CRCs. [BMB Reports 2016; 49(9): 455-456]
\end{abstract}

*Corresponding author. E-mail: kychoi@yonsei.ac.kr

http://dx.doi.org/10.5483/BMBRep.2016.49.9.125

Received 26 July 2016

Keywords: $\beta$-catenin, Colorectal cancer, Ras, Small molecule, Wnt signaling

Abbreviations: APC, adenomatous polyposis coli; CRC, colorectal cancer; EGFR, epidermal growth factor receptor; GSK3 $\beta$, glycogen synthase kinase $3 \beta$; RGS, regulators of G-protein signaling

Perspective to: $\mathrm{Pu}-\mathrm{Hyeon}$ Cha et al., Small-molecule binding of the axin RGS domain promotes $\beta$-catenin and Ras degradation. Nature Chemical Biology (2016) Jun 13. doi: 10.1038/nchembio.2103.
Colorectal cancer (CRC) is one of the most commonly diagnosed cancers and is a major cause of cancer-related deaths throughout the world. Adenomatous polyposis coli (APC) and KRAS mutations are observed in about $90 \%$ and $50 \%$ of human CRC patients, respectively. These mutations lead to aberrant activation of the $\mathrm{Wnt} / \beta$-catenin and Ras pathways which play important and interactive roles during initiation and progression of CRCs. Although these relationships between KRAS and APC mutations in CRC have been illustrated, the mechanism for the cross-talk between the $\mathrm{Wnt} / \beta$-catenin and the Ras pathways is poorly understood. In previous studies, we discovered that Ras was degraded together with $\beta$-catenin upon suppression of $\mathrm{Wnt} / \beta$-catenin signaling (Jeong et al, (2012) Sci Sig 5: ra30, doi: 10.1126/ scisignal.2002242). Inhibition of $\mathrm{Wnt} / \beta$-catenin signaling induced by overexpression of APC or axin resulted in glycogen synthase kinase $3 \beta$ (GSK33)-mediated phosphorylation of Ras at threonine-144 and -148, resulting in polyubiquitin-dependent proteasomal degradation of phosphorylated Ras through recruitment of $\beta-\operatorname{TrCP}$ E3 linker. Both $\beta$-catenin and Ras proteins are frequently co-stabilized in CRCs in both mice and humans. The increased mutant K-Ras and $\beta$-catenin levels that occur concomitantly with $A P C$ loss play critical roles in growth and metastasis of CRC.

Based on these results, we screened a small molecule library and identified KY1220, a compound that was shown in our recent study to induce both $\beta$-catenin and Ras degradations via inhibition of the $\mathrm{Wnt} / \beta$-catenin signaling. By synthesis of its derivatives, we obtained KYA1797K which has improved functionalities in the degradation of both $\beta$-catenin and Ras as well as in suppression of CRC cell proliferation.

We also found that KYA1797K binds to the regulators of G-protein signaling (RGS) domain of axin. Analysis of an axin mutant with an alanine replacement at lysine-147 in the RGS domain revealed that this substitution abolished the effects of KYA1797K on the degradation of both $\beta$-catenin and Ras, indicating that this residue is essential for KYA1797K binding and subsequent destabilization of both $\beta$-catenin and Ras. Furthermore, KYA1797K induced formation of the $\beta$-catenin destruction complex, which activates GSK3 $\beta$, leading to 
phosphorylation of both $\beta$-catenin and Ras (Fig. 1). The $\beta$-TrCP E3-linker was recruited to the phosphorylated $\beta$-catenin and Ras, which were subjected to destabilization via polyubiquitinlation-dependent proteasomal degradation (Fig. 1). This destabilization of $\beta$-catenin and Ras by KYA1797K reduced proliferation of various CRC cells harboring APC and KRAS mutations. In addition, administration of KYA1797K efficiently suppressed tumor growth in mouse xenografts of CRC cells expressing $A P C$ and KRAS mutations and in genetically engineered $A p C^{\mathrm{min} /+} / \mathrm{Kras}^{\mathrm{G} 2 \mathrm{D}} \mathrm{LA} 2$ mice. Collectively, these results indicate that degradation of $\beta$-catenin and Ras by this small molecule-based approach would be a potential strategy for treatment of CRCs with activated $\mathrm{Wnt} / \beta$-catenin and Ras pathways.

Because KYA1797K destabilizes Ras, as well as $\beta$-catenin, our small molecule-based approach may offer a new strategy for the development of Ras-targeting therapeutics. Ras has been considered an attractive target for anti-cancer drug development. Although significant efforts have been made, most of the approaches to control Ras have been unsuccessful. However, the need for Ras-targeting agents is increasing because patients harboring KRAS mutations show unresponsiveness to current therapeutics such as anti-epidermal growth factor receptor (EGFR) agents. Therefore, the small molecules that degrade both $\beta$-catenin and Ras and their improved derivatives may not only be applicable for CRC patients harboring KRAS mutations, but also for those who are unresponsive to the EGFR-targeting therapies due to KRAS mutations.

In conclusion, our small molecule-based approach can be used as a potential strategy for the development of anti-cancer drugs for the treatment of CRC and other cancers with

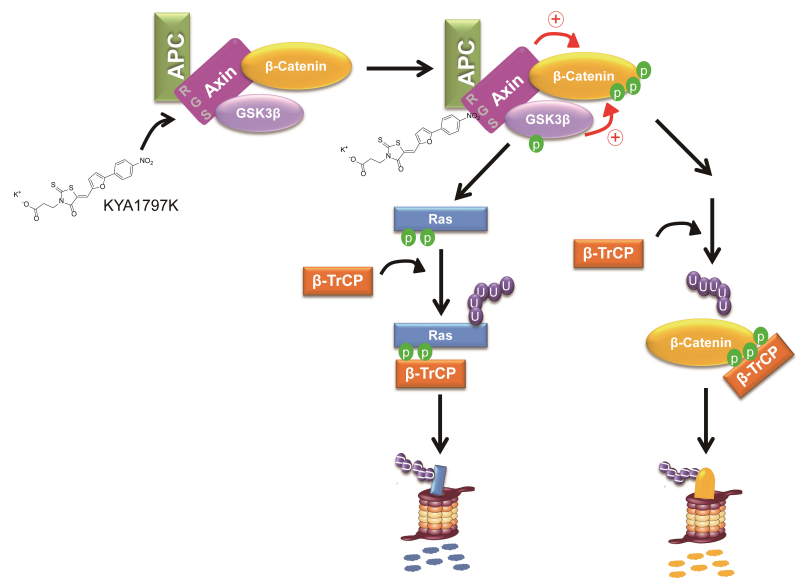

Fig. 1. A Schematic model for the degradation of $\beta$-catenin and Ras by KYA1797K. Binding of KYA1797K with the axin-RGS domain enhances formation of the $\beta$-catenin destruction complex, which activates GSK3 $\beta$. The activated GSK3 $\beta$ phosphorylates both $\beta$-catenin and Ras and these phosphorylated proteins undergo $\beta$-TrCP E3 linker-mediated polyubiquitinylation-dependent proteasomal degradation.

activated $\mathrm{Wnt} / \beta$-catenin and/or Ras pathways.

\section{ACKNOWLEDGEMENTS}

This work was supported by the National Research Foundation of Korea (NRF) funded by the Korean Government (MSIP) (grants 2016R1A5A1004694, 2015R1A2A1A05001873) and a BK21 studentship from the NRF. 